Received: January02,2017

Accepted: January25,2017

Published: February03,2017

\section{New Insights into the Etiopathogenesis of Seborrheic Dermatitis}

\author{
Argir Argirov $^{1}$, Ilko Bakardzhiev ${ }^{2 *}$ \\ ${ }^{1}$ Departmet of Dermatology and Venerology, Medical University of Varna, Bulgaria \\ ${ }^{2}$ Medical College, Medical University of Varna, Bulgaria
}

*Corresponding author: Ilko Bakardzhiev, Medical College, Medical University of Varna, Tsar Osvoboditel 84, Bulgaria, Tel: +359 888768 413; E-mail: varna2008@gmail.com

\section{Abstract}

Seborrheic dermatitis represents a chronic, widespread skin disease that is considered a multifactorial disorder influenced, in part, by Malassezia spp.opportunistic activities as well as by various endogenous and exogenous factors. This review systematizes the recent scientific achievements in the field of insufficiently elucidated etiology and pathogenesis of seborrheic dermatitis.

\section{Keywords:}

Seborrheic Dermatitis;Malassezia; Etiopathogenesis; Immunology; Inflammation; Molecular Biology;

\section{Introduction}

Seborrheic dermatitis is initially described by P. G. Unna in 1887 and the association with Malassezia yeasts is accepted up to the middle of the 20th century, when the observed increased epidermal cell turnover gradually prompts researchers to characterize this condition as being intrinsic to the skin[1,2].

Seborrheic dermatitis is common dermatological problem that affects the seborrheic areas of the body. It is considered the same basic condition like dandruff sharing many features and responding to similar treatments, differing only in locality and severity. Current use of varying terms for seborrheic dermatitis such as sebopsoriasis, seborrheic eczema, dandruff, and pityriasis capitis reflects the complex nature of this common skin pathology [3]. Much controversy remains regarding the pathogenesis of seborrheic dermatitis and its classification in the spectrum of cutaneous diseases as a form of dermatitis, a fungal disease, or an inflammatory disease, closely related with psoriasis.

The prevalence of seborrheic dermatitis peaks when sebaceous gland activity is high, during the first three months of life (infantile seborrheic dermatitis), during puberty and when sebum ex- cretion is reduced after the age of 50 years [2]. Because of smaller sebum production, seborrheic dermatitis flares occur more often in spring than in summer. Disease flare could be associated with altered population dynamics affected not only by variations in sebaceous gland activity but also by modifications in other nutrients supplied by sweat, such as essential amino acids like glycine and tryptophan.

Glycine stimulates the fast growth of M. furfur and after its exhaustion yeast cells employ tryptophan as a nitrogen source, increasing the production of indolic metabolites [4]. Such cycles of population growth, bioactive indole production, and subsequent deprivation of nutrients result in insufficiently masked antigens and ligands on the surface of the yeast cells leading to immune system activation.

Seborrheic dermatitis occurs on the scalp as well as on face, retroauricular area, and the upper chest, causing flaking, scaling, inflammation, pruritus, and, sometimes, marked erythema [5]. Flaking is usually white-to-yellowish, and may be oily or dry. Various intrinsic and environmental factors, such as sebaceous secretions, skin surface fungal colonization, individual susceptibility, and interactions between these factors play a pathogenetic role in seborrheic dermatitis.

\subsection{Etiology of Seborrheic Dermatitis}

There are three principal factors that play a role in the etiology of seborrheic dermatitis [6]: sebaceous gland secretion, alteration in colonization and metabolism of cutaneous microflora (Malassezia spp.), as well as individual susceptibility and host response [7].

Malassezia is a monophyletic genus of fungi found on the skin of seven billion humans and associated with a variety of conditions, including dandruff, seborrheic dermatitis, atopic eczema/dermatitis, pityriasis versicolor, and folliculitis [8]. Recently, this genus represents a hot topic of basic research on taxonomy, physiology, biochemistry, ecology, immunology, and metabolomics [2]. It encompasses a total of 14 species [2, 9]. They are the following: $M$. furfur, $M$. pachydermatis, $M$. sympodialis, M. globosa, M. obtusa, M. restricta, M. slooffiae, M. dermatis, 
M. japonica, M. yamatoensis, M. nana, M. caprae, M. equina, and M. cuniculi.

As Malassezia spp. are present on the skin surface and within layers of the stratum corneum, variations in technique in obtaining specimen and quantifying the organism likely explain the differences in findings among available studies (Gupta AK). M. globosa and $M$. restricta are commensal yeasts requiring an exogenous source of lipids and are most commonly associated with seborrheic dermatitis [10]. They are capable of degrading lipids in sebum with production of free fatty acids and triglycerides, followed by consumption of certain saturated fatty acids. The remaining modified unsaturated short-chain fatty acids are more capable of penetrating the skin and inducing the inflammation [7]. The increase in natural killer cells (NK1+), CD16+ cells and inflammatory interleukins as well as complement activation in lesional skin as compared to non-lesional skin and in skin of healthy controls suggests an augmented inflammatory response in seborrheic dermatitis patients.

Inflammatory markers recorded by immunocytochemistry of skin biopsy specimens from seborrheic dermatitis lesions show an increase in levels of various inflammatory mediators such as interleukin-1 Îś, interleukin-1 Î̌s, interleukin-2, interleukin-4, interleukin-6, interleukin-10, interleukin-12, gamma interferon, and tumor necrosis factor alpha in the epidermis and around the follicles of diseased skin [11].

The immunoenzymatic examination of the concentrations of selected inflammatory factors in 36 seborrheic dermatitis patients, 16 females and 20 males, at a mean age of 38.61Âś13.77 years (range, 19-76 years), and in 30 healthy volunteers, 23 females and seven males, at a mean age of 37.41 Âś6.08 years (range, 24-65 years) demonstrates statistically significantly higher levels of interleukin-2 and gamma interferon in these patients than in the control subjects and proves the role of these cytokines in the pathogenesis of seborrheic dermatitis [12].

Malassezia sp. are a ubiquitous component of the human skin microbiome and are associated with a myriad of skin problems [13]. With the use of modern genomic and systems biology tools, new insights could be gained in the interaction between humans and these eukaryotes which are most intimately associated thus leading to perspectives on the duality of their symbiotic and antagonistic relationship.

The measures at the structure/function level such as epidermal morphology, Malassezia infiltration and instrumental assessments related to moisture content support the basic etiology sequence [14]. They enable a deeper understanding of seborrheic dermatitis pathophysiology by demonstrating that the superficial signs and symptoms result from an irregular epidermal construction and subsequnt disorganization in seborrheic dermatitis onset. The specific molecular mechanisms in the skin establish the inflammatory nature of the disease, the poor synchronization of proliferation and differentiation and the relatively ineffective barrier function. The seborrheic dermatitis measures support the inclusion of this pathology in the broad group of inflammatory dermatoses such as psoriasis, atopic dermatitis and acne presenting with different triggering events but sharing the pathophysiology of inflammation, proliferation and skin barrier impairment [14].
While many of the 4285 genes for biosynthetic enzymes are present, $M$. globosa is the only free-living fungus known to lack a fatty acid synthase gene [15]. With a plethora of lipase genes, M. globosa likely satisfies its lipid requirement by hydrolysis of sebum triglycerides.

The analysis of 5.8S rDNA/ITS2 from Malassezia sp. by polymerase chain reaction amplification and restriction fragment length polymorphism methods demonstrates higher $M$. globosa proportions in non-scalp lesions from severe seborrheic dermatitis subjects in Brazil compared with other areas thus suggesting the closer association of this species with such lesions from areas other than scalp [16].

The non-culture-based epidemiological studies of Malassezia population dynamics in healthy or diseased human skin employing techniques for direct identification and quantification of Malassezia DNA from skin specimens do not reveal any significant difference in the ribosomal DNA sequences of the strains colonizing healthy and affected skin [2]. They indicate that the predominant species are $M$. globosa and $M$. restricta found equally on the skin of practically all humans. The use of multilocus sequencing typing for the screening of pathogenic versus non-pathogenic Malassezia strains could highlight the pathobiology of Malassezia yeasts.

The results from a culture-based study of the presence and density of Malassezia spp. in 60 seborrhoeic dermatitis patients and 70 and healthy controls in Serbia show that Malassezia is reliably more frequent in patients than in controls (in $90 \%$ and $60 \%$ of the cases, respectively; $\mathrm{p}<0,01$ ) [17]. The most common isolaets on patient's lesional skin are M. slooffiae (in 26\%), M. globosa and M. sympodialis (in $17 \%$ of the cases each).

$M$. globosa and $M$. restricta are identified through direct detection of fungal DNA using nested polymerase chain reaction in $87.0 \%$ and $81.5 \%$ of 146 Chinese seborrheic dermatitis patients, respectively, accounting together for more than $50 \%$ of Malassezia spp. [18]. There is co-colonization of two or more Malassezia spp. detected by non-culture-based methods in $82.9 \%$ of these patients.

M. dermatis has been isolated in East Asia (Japan and South Korea), while M. obtusa has been isolated mostly in Sweden, Canada, Iran and Indonesia, and Bosnia and Herzegovina [2].

\subsection{Pathogenesis of Seborrheic Dermatitis}

Recently, a more complete description of seborrheic dermatitis pathophysiology based on the combination of three informational strata - the macro (signs and symptoms), micro (physiological structure and function) and biomolecular stratum is provided [14]. The comprehensive description of 'scalp health' adding the new biomolecular markers to the existing clinical parameters has the following benefits: (i) advances the understanding of the biopathology of the disease; (ii) provides a framework to assess the thoroughness of therapies; (iii) enables the use of other clinical endpoints in addition to the commonly observed signs and symptoms; and (iv) establishes relevant subclinical parameters supplementingthe clinical observations.

The recognition of the role of Malassezia yeasts in seborrheic 
dermatitis pathogenesis is reappraised in the 1980s as the antifungal activity of the multiple treatment regimens is a common denominator for seborrheic dermatitis [19].

The characteristic structural and biomolecular abnormalities associated with seborrheic dermatitis are categorized by the following four sequential pathophysiological phases [20]: Malassezia ecosystem and interaction with the epidermis; initiation and propagation of inflammation; disruption of proliferation and differentiation processes of the epidermis, and physical and functional skin barrier disruption.

Seborrheic dermatitis signs and symptoms are well established mainly through traditional (macro) assessment of the scalp skin condition [14].

Its description at the structural and molecular level is now enabled by new molecular measurement capabilities. The emerging pathophysiological model is based on the established etiological phases of Malassezia metabolism initiating the inflammatory cascade, resulting in scalp skin hyperproliferation and incomplete corneocyte differentiation that yields an impaired stratum corneum barrier [14].

Extensive clinical populations using expert flake assessment to identify seborrheic dermatitis are evaluated for a wide range of molecular level biomarkers comparing this pathology and normal populations [21, 22]. The cell envelope protein involucrin as a structural biomarker related to differentiation shows only small differences between the two populations and the keratins 1, 10 and 11 are substantially reduced in the seborrheic dermatitis population indicating incomplete terminal differentiation. Molecular markers of barrier integrity are quantified that complete the etiologic cascade: human serum albumin and the epidermal intercellular lipids [14]. In future, biomarker quantifications associated with reactive oxygen species defense can be identified, as there are indications that oxidative damage either accompanies, or causes seborrheic dermatitis.

Seborrheic dermatitis results from a nonspecific immune response to Malassezia yeasts. They demonstrate an ability to induce immune reactions, depending on the species, the culture growth phase, yeast cell viability, and the integrity of Malassezia cells [23]. M. globosa and M. restricta commonly isolated from human skin present with distinct profiles of proinflammatory cytokine production from epidermal cells, with $M$. globosa stimulating the production of significantly more cytokines than $M$. restricta. Even the use of different culture media results in different compositions of the lipid layer that covers Malassezia cell wall leading to a variable immune system modulation [23]. The production of phospholipases and the response to $\beta$-endorphin is associated with seborrheic dermatitis pathogenesis, too. There is an increased production of phospholipase after $\beta$-endorphin stimulation in pathogenic $M$. pachydermatis strains. This also applies to lipophilic Malasseziaspecies, although it is reproducible in vitro only for M. furfur [24].

Sebum production is increased by Îš-endorphin [25] and a functional Iij-opioid receptor in pathogenic and non-pathogenic M. pachydermatis strains is demonstrated [26]. This points towards the existence of an equivalent sensory pathway in the lipophilic Malassezia species that assists in yeast preparation for a better utilization of sebaceous lipids. The aberrant production of Malassezia phospholipases on the skin leads to epidermal lipid removal, epidermal barrier function disruption, and seborrheic dermatitis development when sebum production is constitutionally decreased.

Malassezia species are identified by phenotypic and genotypic methods and a phylogenetic analysis using Bayesian inference among 134 individuals including subjects without lesions, seborrheic dermatitis patients, HIV positive patients, and HIV patients with seborrheic dermatitis in Colombia [27]. M. restricta is the most frequent species of Malassezia related to seborrheic dermatitis development. Age and gender are not predisposing factors for this disease.

Malassezia speies are implicated in skin diseases including seborrheic dermatitis, atopic dermatitis, pityriasis versicolor and Malassezia folliculitis and act act as an exacerbating factor in and atopic dermatitis [9]. The fatty acids generated by Malassezia lipase induce skin inflammation leading to seborrheic dermatitis development.

The analysis of bacterial microbiotas at lesional and nonlesional sites of 24 seborrheic dermatitis patients by pyrosequencing and quantitative polymerase chain reaction shows that Acinetobacter, Corynebacterium, Staphylococcus, Streptococcus and Propionibacterium are abundant at both sites while Acinetobacter, Staphylococcus and Streptococcus predominate at lesional sites [28]. As these abundant bacteria hydrolyze sebum, they may also contribute to seborrheic dermatitis development.

The presence of host susceptibility factors permitting the transition of Malassezia furfur to its pathogenic form may be associated with immune response and inflammation [3]. Metabolites produced by Malassezia spp. e.g. oleic acid, malssezin, and indole-3carbaldehyde are implicated, too.

Malassezia yeasts, androgens, sebum levels and immune response play important roles in seborrheic dermatitis development while additional factors such as drugs, winter temperatures and stress may exacerbate this disease [29]. Sebaceous glands contribute to skin barrier function and their alteration may play a role in the pathogenesis of seborrheic dermatitis and other common skin diseases such as acne vulgaris, atopic dermatitis, psoriasis, and rosacea [30]. There is a significant increase in the levels of the biological markers cathepsin S, proteinase-activated receptor 2 and histamine patients with seborrheic dermatitis which correlate with clinical parameters [31].

The role of oxidative stress for the etiopathogenesis of seborrheic dermatitis is comparatively examined in 54 patients and 54 healthy volunteers [32]. Serum total antioxidant status and total oxidative status are measured, and oxidative stress index is calculated. Mean total antioxidant status values are significantly lower $(\mathrm{p}=0.024)$ while total oxidative status and oxidative stress index are higher in the patients than in the controls $(\mathrm{p}<0.05)$.

\section{Conclusion}

Further clarification of the interdisciplinary nature of seborrheic dermatitis etiopathogenesis requires purposeful research using contemporary genetic, molecular-biologic and immunohistochemical methodology. 


\section{References}

1. Unna PG. Seborrheic eczema. J Cutan Dis. 1887;5:449-453.

2. Gaitanis G, Magiatis P, Hantschke M, Bassukas ID, Velegraki A. The Malassezia genus in skin and systemic diseases. Clin Microbiol Rev. 2012;25(1):106-141. doi: 10.1128/CMR.00021-11.

3. Dessinioti C, Katsambas A. Seborrheic dermatitis: etiology, risk factors, and treatments: facts and controversies. Clin Dermatol. 2013;31(4):343-351. doi: 10.1016/j.clindermatol.2013.01.001.

4. Barchmann T, Hort W, Krämer HJ, Mayser P. Glycine as a regulator of tryptophan-dependent pigment synthesis in Malassezia furfur. Mycoses. 2011;54(1):17-22. doi: 10.1111/j.14390507.2009.01758.x

5. Borda LJ, Wikramanayake TC. Seborrheic dermatitis and dandruff: A comprehensive review. J Clin Investig Dermatol. 2015;3(2). doi: 10.13188/2373-1044.1000019.

6. Del Rosso JQ. Adult seborrheic dermatitis: a status report on practical topical management. J Clin Aesthet Dermatol. 2011;4(5):32-38.

7. Picardo M, Cameli N. Seborrheic dermatitis. In: EvidenceBased Dermatology. Williams H, Bigby M, Diepgen T, Herxheimer A, Naldi L, Rzany B, eds. 2nd ed. Malden, MA, Blackwell Publishing, 2008;164-170.

8. Ashbee HR, Scheynius A. Malassezia. In: Ashbee HR, Bignell EM, eds. Pathogenic yeasts. The yeast handbook. Berlin and Heidelberg, Springer-Verlag. 2010;209-230.

9. Harada K, Saito M, Sugita T, Tsuboi R. Malassezia species and their associated skin diseases. J Dermatol. 2015;42(3):250-257. doi: 10.1111/1346-8138.12700.

10. Gupta AK, Bluhm R, Cooper EA, Summerbell RC, Batra R. Seborrheic dermatitis. Dermatol Clin. 2003;21(3):401-412.

11. Faergemann J, Bergbrant IM, DohséM, Scott A, Westgate G. Seborrhoeic dermatitis and Pityrosporum (Malassezia) folliculitis: characterization of inflammatory cells and mediators in the skin by immunohistochemistry. Br J Dermatol. 2001;144(3):549556.

12.Trznadel-Grodzka E, Błaszkowski M, Rotsztejn H. Investigations of seborrheic dermatitis. Part I. The role of selected cytokines in the pathogenesis of seborrheic dermatitis. Postepy Hig Med Dosw (Online). 2012;66:843-847. doi: $10.5604 / 17322693.1019642$.

13. Saunders CW, Scheynius A, Heitman J. Malassezia fungi are specialized to live on skin and associated with dandruff, eczema, and other skin diseases. PLoS Pathog. 2012;8(6):e1002701. doi: 10.1371/journal.ppat.1002701.

14. Schwartz JR, Messenger AG, Tosti A, Todd G, Hordinsky M, Hay RJ, et al. A comprehensive pathophysiology of dandruff and seborrheic dermatitis - towards a more precise definition of scalp health. Acta Derm Venereol. 2013;93(2):131-137. doi: 10.2340/00015555-1382.

15. Xu J, Saunders CW, Hu P, Grant RA, Boekhout T, Kuramae EE, et al. Dandruff-associated Malassezia genomes reveal convergent and divergent virulence traits shared with plant and human fungal pathogens. Proc Natl Acad Sci USA. 2007;104(47):1873018735.

16. Soares RC, Zani MB, Arruda AC, Arruda LH, Paulino LC. Malassezia intra-specific diversity and potentially new species in the skin microbiota from Brazilian healthy subjects and seborrheic dermatitis patients. PLoS One. 2015;10(2):e0117921. doi: 10.1371/journal.pone.0117921.

17. Barac A, Pekmezovic M, Milobratovic D, Otasevic-Tasic S, Radunovic M, Arsic Arsenijevic V. Presence, species distribution, and density of Malassezia yeast in patients with seborrhoeic dermatitis - a community-based case-control study and review of literature. Mycoses. 201;58(2):69-75. doi: 10.1111/myc.12276

18. Zhang H, Ran Y, Xie Z, Zhang R. Identification of Malassezia species in patients with seborrheic dermatitis in China. Mycopathologia. 2013;175(1-2):83-89. doi: 10.1007/s11046012-9606-z

19. Shuster S. The aetiology of dandruff and the mode of action of therapeutic agents. Br J Dermatol. 1984;111(2):235-242.

20. Saint-Leger D. Dandruff (pityriasis capitis simplex): of yeasts and men. In: The science of hair care. Bouillion C, Wilkinson J, eds. 2nd edn. New York, Taylor \& Francis. 2005;609-631.

21. Kerr K, Darcy T, Henry J, Mizoguchi H, Schwartz J, Morrall $\mathrm{S}$, et al. Epidermal changes associated with symptomatic resolution of dandruff: Biomarkers of scalp health. Intl J Derm. 2011;50(1):102-113. doi: 10.1111/j.1365-4632.2010.04629.x.

22. Kerr K, Schwartz J, Filloon T, Fieno A, Wehmeyer K, Szepietowski JC, et al. Scalp stratum corneum histamine levels: Novel sampling method reveals correlation to itch resolution in dandruff/seborrheic dermatitis. Acta Derm Venereol. 2011;91(4):404-408. doi: 10.2340/00015555-1073

23. Thomas DS, Ingham E, Bojar RA, Holland KT. In vitro modulation of human keratinocyte pro- and anti-inflammatory cytokine production by the capsule of Malassezia species. FEMS Immunol Med Microbiol. 2008;54(2):203-214. doi: 10.1111/j.1574-695X.2008.00468.x 
24. Vlachos C, Gaitanis G, Alexopoulos EC, Papadopoulou C, Bassukas ID. Phospholipase activity after $\beta$-endorphin exposure discriminates Malassezia strains isolated from healthy and seborrhoeic dermatitis skin. J EADV. 2013;27(12):1575-1578. doi: 10.1111/j.1468-3083.2012.04638.x

25. Zouboulis CC, Baron JM, Böhm M, Kippenberger S, Kurzen $\mathrm{H}$, Reichrath $\mathrm{J}$, et al. Frontiers in sebaceous gland biology and pathology. Exp Dermatol. 2008;17(6):542-551. doi: 10.1111/j.1600-0625.2008.00725.x.

26. Cafarchia C, Dell'Aquila ME, Traversa D, Albrizio M, Guaricci AC, de Santis T, et al. Expression of the micro-opioid receptor on Malassezia pachydermatis and its effect in modulating phospholipase production. Med Mycol. 2010;48(1):73-78. doi: $10.3109 / 13693780902718347$.

27. Amado Y, Patiño-UzcÃątegui A, Cepero de García MC, Tabima J, Motta A, CÃąrdenas M, et al. Seborrheic dermatitis: predisposing factors and ITS2 secondary structure for Malassezia phylogenic analysis. Med Mycol. 2013;51(8):868-875. DOI: 10.3109/13693786.2013.820001

28. Bukvić Mokos Z, Kralj M, Basta-JuzbašićA, Lakoš Jukić I. Seborrheic dermatitis: an update. Acta Dermatovenerol Croat.
2012;20(2):98-104.

29. Tanaka A, Cho O, Saito C, Saito M, Tsuboi R, Sugita T. Comprehensive pyro sequencing analysis of the bacterial microbiota of the skin of patients with seborrheic dermatitis. Microbiol Immunol. 2016;60(8):521-526. doi: 10.1111/1348-0421.12398.

30. Shi VY, Leo M, Hassoun L, Chahal DS, Maibach HI, Sivamani RK. Role of sebaceous glands in inflammatory dermatoses. J Am Acad Dermatol. 2015;73(5):856-863. doi: 10.1016/j.jaad.2015.08.015.

31. ViodÂl' C, Lejeune O, Turlier V, Rouquier A, Casas C, Mengeaud V, et al. Cathepsin S, a new pruritus biomarker in clinical dandruff/seborrhoeic dermatitis evaluation. Exp Dermatol. 2014;23(4):274-275. doi: 10.1111/exd.12357.

32. Emre S, Metin A, Demirseren DD, Akoglu G, Oztekin A, Neselioglu S, et al. The association of oxidative stress and disease activity in seborrheic dermatitis. Arch Dermatol Res. 2012;304(9):683-687. doi: 10.1007/s00403-012-1254-0 